\title{
Corela
}

Cognition, représentation, langage

6-2 | 2008

Vol. $6, n^{\circ} 2$

\section{Phénomènes de discordance entre morphologie et syntaxe : de la zizanie dans le collectif !}

\section{Blandine Pennec}

\section{OpenEdition}

\section{Journals}

Édition électronique

URL : http://journals.openedition.org/corela/274

DOI : $10.4000 /$ corela. 274

ISSN : $1638-573 \mathrm{X}$

\section{Éditeur}

Cercle linguistique du Centre et de I'Ouest - CerLICO

Référence électronique

Blandine Pennec, «Phénomènes de discordance entre morphologie et syntaxe : de la zizanie dans le collectif ! », Corela [En ligne], 6-2 | 2008, mis en ligne le 15 décembre 2008, consulté le 19 avril 2019. URL : http://journals.openedition.org/corela/274 ; DOI : 10.4000/corela.274

Ce document a été généré automatiquement le 19 avril 2019

\section{(c) (i) (2)(2)}

Corela - cognition, représentation, langage est mis à disposition selon les termes de la licence Creative Commons Attribution - Pas d'Utilisation Commerciale - Partage dans les Mêmes Conditions 4.0 International. 


\title{
Phénomènes de discordance entre morphologie et syntaxe : de la zizanie dans le collectif!
}

\author{
Blandine Pennec
}

Tous nos remerciements vont à Jean-Louis Trouillon pour son précieux travail de collecte d'exemples, ainsi que pour la minutieuse relecture de ce document.

1 Certaines grammaires ${ }^{1}$, dans leurs chapitres consacrés à l'étude des catégories de fonctionnement des noms, mentionnent le fait qu'en langue anglaise, il existe des termes présentant un écart entre leur morphologie et l'accord qu'ils suscitent. Une morphologie caractéristique d'un singulier peut ainsi donner lieu à un accord au pluriel, tandis qu'un nom portant morphologiquement la marque du pluriel peut en revanche donner lieu à un accord au singulier. Ces phénomènes de non-coïncidences sont relativement fréquents en langue anglaise, à tel point qu'ils en constituent probablement un trait distinctif. Ces cas sont d'autant plus intéressants qu'ils semblent répondre à un principe selon lequel la signification reprend ses droits, devenant alors prédominante par rapport aux contraintes morphologiques.

2 De fait, cette étude se donne pour objectif de présenter méthodiquement et de tâcher d'expliquer ces phénomènes de discordance entre morphologie et syntaxe, sachant qu'ils dépassent amplement le cadre des exemples généralement cités par les grammaires ${ }^{2}$. En conséquence, nous essaierons de remettre un peu d'ordre dans cette "zizanie » qui semble caractériser les phénomènes d'accord en langue anglaise.

\section{Les noms dits « collectifs"}

\subsection{Fonctionnement au pluriel et accord sylleptique}

3 Littéralement, un nom peut être qualifié de «collectif » lorsque le terme en question réfère à un ensemble d'éléments (ex : party, government, humanity, mankind). Afin de mieux comprendre l'emploi de ces termes, rappelons quelques principes généraux concernant 
les catégories de fonctionnement des noms. Reprenons à cette fin la catégorisation effectuée dans la théorie des opérations énonciatives, qui distingue trois catégories de fonctionnement: celles du discret, du continu dense et du continu compact. Selon la définition donnée par Antoine Culioli ${ }^{3}$, discret, dense et compact correspondent à des types de pondération différents, que l'on peut représenter ainsi :

\begin{tabular}{|l|l|l|}
\hline Qnt Qlt & (Qnt) Qlt ${ }^{4}$ & Qlt \\
\hline discret & continu dense & continu compact \\
\hline
\end{tabular}

4 Ainsi, les noms discrets renvoient à des éléments que l'on peut dénombrer, autrement dit isoler en unités distinctes (ex : chair), les noms fonctionnant en continu dense peuvent être dénombrés uniquement grâce à l'emploi de dénombreurs (ex: luggage, dénombré à l'aide de $a$ piece of) et les continus compacts ne sont pas naturellement dénombrables, puisqu'ils renvoient à un tout difficilement sécable (ex : humility). Ceci explique, dans la représentation ci-dessus, que les termes discrets et continus denses fassent intervenir, outre une dimension qualitative, une dimension quantitative. Les continus compacts, que l'on ne peut pas dénombrer, font en revanche uniquement intervenir une dimension qualitative.

5 Si l'on en revient aux noms à sens collectif, et que l'on applique cette classification, on distingue bien trois ensembles :

- les collectifs fonctionnant en continu dense, du type furniture ou luggage,

- les collectifs en continu compact, du type mankind ou humanity,

- les collectifs fonctionnant discontinu/ discret, du type party ou government.

6 Sauf emploi de dénombreurs, la première catégorie donne systématiquement lieu à un accord au singulier (ex: Reassuringly, your luggage is carried by car between each stop), la seconde donne également lieu à un accord au singulier (ex: Mankind is a dominant species because it's adaptable), tandis que la troisième catégorie peut, de façon surprenante pour un francophone, donner lieu à un accord au pluriel (ex: The Government are not contributing.)

7 Cette possibilité d'un accord syntaxique au pluriel peut être désignée sous le nom d'« accord sylleptique », ce qui signifie qu'il y a accord, non pas avec la forme du nom, mais avec son sens. Puisque le terme réfère à un groupe d'individus, la saisie peut en effet être opérée sous un angle dissociatif et additif: on envisage chacun des membres constituant ce groupe, et le référent associé au nom est alors perçu comme la totalité de ses parties. Parmi les différentes catégories de termes à sens collectif, ceux qui sont compatibles avec cet accord sylleptique sont d'ailleurs les seuls auxquels les grammaires et dictionnaires réservent l'appellation « noms collectifs ».

Cet accord au pluriel se retrouve dans le cas d'un certain nombre de termes que nous allons, sans prétendre à l'exhaustivité, tenter de recenser de façon organisée. Certains de ces termes sont fréquemment cités par les grammaires, mais ce n'est pas nécessairement le cas de tous les noms ci-dessous. Dans la suite de cette étude, nous essaierons d'expliquer comment prédire la compatibilité d'un terme avec l'accord sylleptique.

Remarquons en premier lieu que les termes caractérisés par ce type de discordance réfèrent à : 
$\rightarrow$ des groupes institutionnalisés de personnes:

Il peut, tout d'abord, s'agir de termes appartenant au champ sémantique des activités politiques:

\section{- government ${ }^{5}$ :}

(1) Neil O'Brien, of the Open Europe campaign group, said: "This report shows again that it is a significant transfer of power, that the Government have not been honest." (The Daily Telegraph online)

11 Similairement à government, citons son antonyme:

\section{- opposition:}

(2) The opposition know exactly what they're doing. We don't. (The Scotsman)

12 Un terme sémantiquement connexe aux précédents, le terme party, présente le même type de fonctionnement :

- party:

(3) Led by parish councillor Jan Dyer who has spent two 'very successful days'; working with fellow councillors and other people from the village at the pond site, the working party are now waiting until the pond is dredged before carrying out further work. (BNC C88 1501)

13 L'accord sylleptique caractérisant ici working party se retrouve naturellement lorsqu'il s'agit de political party. Il est en outre notable que les termes hyponymes ${ }^{6}$ de political party peuvent présenter un fonctionnement similaire. Il en est ainsi du terme figurant dans l'exemple suivant, le terme Labour (rappelons qu'inversement, il serait possible de dire que party est un hyperonyme relativement à Labour) :

\section{- Labour:}

(4)The problem with New Labour is that they are not new and they are not Labour enough. Compass is calling for Labour to use the Scottish defeat as an opportunity to try and make the political weather, not just find the best place to shelter from it." (The Scotsman)

14 Les termes donnant lieu à un accord sylleptique peuvent, d'autre part, appartenir au champ sémantique des forces de l'état:

- police:

(5) If the police hope to gain the trust of the people of Broadwater Farm, they must begin by showing good faith towards those that the residents have chosen to represent themselves; the local council and the community organisations here. (BNCA2J 242)

- army:

(6) And the Army are bound to be establishing other water points. (BNC AN71628)

Il est notable que l'un des méronymes ${ }^{7}$ du terme army, à savoir battalion, présente le même type de fonctionnement :

\section{- battalion:}

(7) It was only in February that I visited the battalion, with their families, to wish them God speed before their deployment. (The Prince of Wales)

Un même type d'accord caractérise l'antonyme des termes que nous venons de citer :

- enemy:

(8) At least the enemy are in a similar situation probably much worse off then we are judging by the amount of shells passing over our heads to fall in their positions. (BNC A611630)

$\rightarrow$ des groupements de personnes dans le cadre du travail : 
17 L'accord sylleptique est également suscité par des termes évoquant le monde du travail, plus précisément celui des entreprises et industries. Cette fois encore, c'est un ensemble de relations tout-parties qui se dessine. En effet, nous trouvons en premier lieu des termes qui réfèrent à des groupements d'industries. Tel est le cas des termes consortium et federation tels qu'ils sont illustrés ci-dessous :

\section{- consortium:}

(9) The ongoing takeover battle between Scottish \& Newcastle and Carlsberg is becoming increasingly ill tempered. The consortium of Carlsberg and Heineken yesterday upped their offer for the Edinburgh brewer from 720p per share to 750p, an offer that was swiftly rejected. S\&N branded the offer 'wholly inadequate' while the Danish/Dutch bidders claimed they were 'very disappointed by the tone and speed of S\&N's response.' (The Scotsman)

\section{- federation:}

(10) However, the Federation of Small Businesses Scotland weren't so supportive, with spokesman Andrew Watson sounding a cautionary note that over strident advocacy could prove counter-productive. (The Scotsman)

Les méronymes correspondant à ces deux termes présentent un fonctionnement similaire :

- firm :

(11) I hope the plumber's firm are suitably ashamed of themselves. (BNC CBC 9894)

- company :

(12) If the publishing company are actively involved in acquiring a recording contract for the artist, they would provide studio time for producing demos. (BNC A6A 1328)

19 Par une autre relation de méronymie (relative à firm et company), on obtient ensuite des termes référant au personnel de ces entreprises, et qui présentent eux aussi la possibilité d'un accord sylleptique :

- staff:

(13) Standard Life staff are braced for what many fear will be job cuts after a number of workers were called to meetings in Edinburgh today. Speculation suggests that independent financial adviser support staff could be affected by job losses, though the life giant has refused to be drawn on the reasons for calling the meetings. (The Scotsman)

- management:

(14) Prudential staff in Stirling could be set to learn the fate of their jobs this morning. Some 1,000 jobs at the life insurance giant's Craigforth base in Stirling are believed to be in danger of outsourcing as the company attempts to cut costs by up to $£ 195$ million. Senior management were called to a meeting last night and it is expected that Prudential will make a statement to the city this morning. (BBC Scotland online)

\section{- committee:}

21 (15) 'The committee are absolutely appalled'; he said, 'particularly in view of a directive sent to clubs in regard to foul play.' (BNC A22 89)

$\rightarrow$ des groupements de personnes dans le cadre d'activités communes :

Le regroupement peut s'effectuer dans le cadre d'activités sportives :

- team:

(16) As soon as he is back his team are around him, removing the protective plates and helmet in order to ease the discomfort for a while. (BNC A77 1392)

Il peut également s'agir d'un regroupement dans le cadre de spectacles :

- audience: 
(17) The audience are coming to these shows knowing fully that they should be prepared to deal with the present and the future. (BNC C9K 1533)

\section{fonctionnement similaire :}

- public:

(18) I would agree that the game is played by professional sportsmen, but they are only "professional", because the public are prepared to pay to watch them. (BNC BN9 1296)

Le groupement de personnes peut s'effectuer dans le cadre d'activités religieuses :

\section{- congregation:}

(19) That the Free Church congregation are burdened with the expense of maintaining a place of Worship, while they have no minister to occupy it, Sermon being held there only during two Sabbaths for upwards of three months. (BNC FTT 1586)

Un terme connexe au précédent, mais évoquant cette fois une activité religieuse très précise, présente un fonctionnement similaire (bien que de façon plus rare). Il s'agit du terme procession, illustré par l'exemple suivant :

\section{- procession :}

(20) The songs are good, and the Procession are not afraid of immersing themselves totally in their music, but perhaps a more measured approach might be worthwhile. (BNC K4Y994)

L'activité permettant le regroupement est un peu plus diffuse avec le terme crowd, mais nous avons tout de même affaire à un rassemblement de personnes qui marchent ou attendent un événement :

- crowd:

(21) The vast crowd are allowed to walk freely in this area. (BNC AJA 287)

Enfin citons un terme extrêmement générique qui a la particularité de ne pas évoquer par lui-même le type d'activité regroupant les personnes en question :

\section{- group:}

(22) The group - who include union bosses, executive officials, and MPs - fear their traditional support will defect as the SNP is seen to fill the vacuum on the left. (The Scotsman)

$\rightarrow$ des groupements de personnes dans le cadre familial :

\section{- family:}

(23) The family are beginning to bubble again and we stop and chat happily to the old couple. (BNC CA9 1822)

\section{- couple:}

(24) Pink sands and towering granite boulders dot the beach, which is sheltered by a reef and the calm and shallow waters in a gorgeous setting will make a couple feel they are in the Garden of Eden. (Ezine)

$\rightarrow$ des groupements d'animaux, sous un angle littéral ou métaphorique :

- cattle (sens littéral):

(25) Sheep flocks are set stocked and are dependent on pasture the year round apart from a limited provision of fodder in times of storm or supplementary food in late pregnancy while cattle are customarily fed during winter. (BNC ALG 50)

- fry (sens littéral) :

(26) The fry are extremely tiny, too small to take even Artemia nauplii for the first 5-6 days. (BNC C95 786)

- flock (sens biblique, métaphorique) : 
(27) The flock are unlikely to have been as completely innocent as the latter suggests, and their guilt may well have had a secular aspect, for it might be doubted whether Cnut would have become involved with spiritual shortcomings. (BNC HXX 1483) présentent en réalité une alternance de fonctionnement et peuvent ainsi, dans d'autres énoncés, être suivis d'un verbe ou d'un adjectif possessif au singulier. Citons, à titre d'exemples, des termes caractérisés par des accords extrêmement fluctuants :

\section{- family:}

(28) The family is a microcosm of social existence for which our young are preadapted. (BNC B10278)

\section{- team:}

(29) The second person then follows so that in the end the whole team is linked together by string. (BNC C8P 1074)

\section{- government:}

(30) The Institute of Fiscal Studies has warned that the government has delayed a necessary 'fiscal repair job' until after the next election. (The Scotsman)

\section{- company:}

(31) Small wonder that the Italian company is keen on merging. (BNC ABH 2248) première vue surprenante, s'explique en réalité par une question de saisie du référent lorsque le référent est perçu de façon externe, dans sa globalité et son unité, l'accord peut être au singulier. En revanche, lorsque le référent est perçu de façon interne, sous l'angle de la pluralité - et éventuellement de l'hétérogénéité - de ses composants, alors l'accord peut être au pluriel. L'accord de ce type de noms est finalement le reflet d'un choix - plus ou moins conscient - de l'énonciateur. Les paramètres co-textuels se présentent parfois comme des indices du choix opéré, soit en faveur d'une cohésion maximale, ou au contraire d'une relative dissociation des éléments. Ainsi, si l'on reprend l'exemple comportant family cité un peu plus haut, on se rend compte que l'énonciateur établit, par le biais de la copule be, une équivalence entre le référent de family et celui de microcosm : la saisie est donc globale, et la famille n'est plus perçue sous l'angle de ses composants, mais elle apparaît comme un lieu : ce sont ainsi des propriétés nouvelles qui sont activées, propriétés qui relèguent au second plan ses composants humains.

Considérons maintenant l'exemple faisant intervenir le nom team : le prédicat link together y est évocateur d'une unité, ce qui est tout à fait cohérent avec la saisie reflétée par l'accord au singulier. Si l'on examine ensuite l'énoncé employant government, les indices co-textuels permettant d'expliquer la saisie unitaire ne sont pas aussi manifestes que dans les deux autres exemples, mais le sens global de la phrase est cohérent avec une saisie unitaire car cet exemple réfère à une décision (et donc à un point d'aboutissement 
commun) du gouvernement. Il en va de même dans l'énoncé employant company où l'on réfère cette fois encore à une décision commune, résultante.

\subsection{Précisions : les propriétés nominales favorisent l'accord sylleptique de termes au singulier}

Afin qu'un nom dont la morphologie correspond à un singulier puisse donner lieu à un accord au pluriel, nous avons mentionné plus haut la nécessité que ce nom soit à la fois discret et collectif. Ceci explique pourquoi, en dépit d'une signification faisant également intervenir des sous-unités multiples, des termes fonctionnant en continu dense tels que hair, luggage, furniture, evidence ou encore rubbish donnent systématiquement lieu à un accord au singulier. Bien que leur fonctionnement soit, sur le plan référentiel, similaire à celui de party ou government, la langue organise toutefois une saisie différente de la réalité dans l'un et l'autre cas. En effet, en ce qui concerne hair, luggage, etc...les composants ne sont pas construits (du moins par la langue) comme isolables, mais ils forment une totalité envisagée dans sa continuité. Cette saisie est, soulignons-le, le propre de la langue, car la réalité extralinguistique permettrait, elle, d'isoler des cheveux ou des meubles. On rappelle d'ailleurs à ce propos la différence de saisie entre la langue anglaise et la langue française qui, elle, fait de « cheveu » ou de «meuble » des termes discrets. Il est en outre à noter que ces termes présentant un fonctionnement en continu dense (et ne favorisant donc pas l'accord sylleptique) sont généralement des termes référant à des inanimés, à l'inverse des collectifs discrets mentionnés plus haut, référant soit à des animés humains, soit à des animaux. Cette caractéristique peut fonctionner comme un indice permettant d'établir plus aisément une ligne de partage entre les deux catégories de termes.

Concernant maintenant les termes fonctionnant en continu compact, tels que humanity ou mankind, le problème est différent car ils réfèrent pour leur part à un ensemble d'animés humains. Ils y réfèrent toutefois sous un angle purement qualitatif : c'est l'essence même de l'humanité qui est prise en compte, et non ses composants. On dit ainsi de ces termes qu'ils réfèrent à la classe, non pas en extension, mais en intension. Corrélativement, ceci explique l'accord au singulier qui caractérise systématiquement ces termes, les faisant ainsi contraster avec les noms collectifs discrets.

\section{Les expressions $N 1$ of $N 2$ à sens collectif}

Parallèlement aux noms collectifs proprement dits, dont nous avons esquissé plus haut un recensement, il existe un certain nombre de termes, eux aussi discrets et à sens collectif, qui peuvent également donner lieu à un accord sylleptique, à la condition qu'ils soient employés au sein d'expressions N1 of N2. Employés seuls (du moins lorsque cela est possible), ces termes suscitent nécessairement un accord au singulier, mais lorsqu'ils correspondent au N1 d'une structure $\mathrm{N} 1$ of N2, ils présentent alors une alternance de fonctionnement, autrement dit une alternance d'accords au singulier et au pluriel (ils sont alors à rapprocher des noms collectifs à proprement parler). Il s'agit de termes tels que a set (of), a succession (of,) a queue (of)....termes dont la typologie a été effectuée par V. Arigne $^{8}$. En complémentarité avec ce travail, nous allons ici envisager ces termes sous l'angle de l'accord qu'ils peuvent susciter. 
Mentionnons tout d'abord que ces noms, dont nous empruntons la liste à V. Arigne, peuvent selon nous être divisés en trois catégories principales :

$\rightarrow$ La première correspond à des regroupements simples d'occurrences. Cette catégorie est constituée par des termes tels que a group, a set, a batch, a bundle, a bunch + $\mathrm{OF}+\mathrm{N} 2$.

$\rightarrow$ La deuxième catégorie correspond à des regroupements d'occurrences selon des principes organisateurs, qui peuvent être :

- le temps : a succession, a sequence, a series + OF + N2,

- le mouvement ou l'absence de mouvement : a queue, a convoy, a volley + OF + N2,

- une disposition verticale ou horizontale: a stack, a pile, an array, a battery, + OF + N2,

- des critères de sélection qualitatifs: an assortment, a selection, a choice, a spectrum, a variety, a list, a catalogue $+\mathrm{OF}+\mathrm{N} 2$.

Si l'on revient précisément à l'objet de cette étude, à savoir le type d'accord syntaxique suscité par ces termes, les observations effectuées à l'aide du British National Corpus montrent que chacun d'entre eux peut, au sein de structures $N 1$ of $N 2$, donner lieu, soit à un accord au singulier, soit à un accord au pluriel. Fournissons quelques exemples pour chacune des catégories recensées plus haut, exemples que nous commenterons dans le but de dégager des tendances permettant de prédire le type d'accord.

$\rightarrow$ Groupements simples :

Mis à part le cas du terme group, qui est un nom collectif à proprement parler (cf. paragraphe précédent) et peut donc, même lorsqu'il est employé seul, donner lieu à une alternance singulier/ pluriel, les deux autres termes, à savoir set et batch s'accordent théoriquement au singulier uniquement. L'alternance est toutefois possible lorsqu'ils sont employés en tant qu'expressions à valeur quantificatrice, ainsi qu'on l'observe dans les exemples suivants, employant le terme set:

(32) Another critical prerequisite is the existence of $a$ set of external conditions that makes the choice of maintaining the status quo either unlikely or impossible. (BNC FA8 378)

Cet exemple, illustrant un cas typique d'accord au singulier, contraste fortement avec :

(33) Despite their focus on resilience, a set of Sequent's customers are planning to extend their clusters beyond this and towards transparent data sharing. (BNC CPE 100)

41 C'est cette fois un accord au pluriel qui est à l'œuvre, contrairement aux attentes suscitées par l'une des règles élémentaires de la syntaxe, selon laquelle, dans une structure N1 of N2, l'accord s'effectue avec N1 (un exemple typique est: One of my friends was at Sherborne and speaks very posh). Dans l'exemple employant set, deux explications peuvent être avancées :

- soit l'on peut considérer que l'accord s'effectue avec N2,

- soit l'on peut envisager que N1 acquiert un fonctionnement de type «nom collectif » en raison de sa complémentation par N2. En effet, si l'on examine le cas de set employé seul, on constate qu'il diffère d'un nom collectif proprement dit (par exemple de government) en ce qu'il n'indique pas par lui-même la nature des unités qui le composent (c'est un terme générique qui peut être complémenté par divers types de N2). Lorsqu'une complémentation est effectuée, nous avons alors accès à la nature des N2, et l'accord sylleptique devient finalement possible.

Il existe, comme ici, des exemples pour lesquels il n'est pas possible de pencher en faveur de l'une ou l'autre hypothèse. Nous allons voir toutefois que certains énoncés relèvent de 
façon manifeste de l'une des deux explications. Pour ce faire, examinons les autres catégories mentionnées plus haut :

$\rightarrow$ Groupements d'occurrences selon des principes organisateurs :

- le temps:

On observe, cette fois encore, la possibilité d'une alternance :

(34) A succession of flowers is the life-blood of most gardens. (BNC CABAK 61520 )

(35) A succession of inspectors visit estates before recommending awards. (BNC

K97 12551)

Tout comme dans la paire citée plus haut (occurrences de set), ces exemples illustrent un accord au singulier lorsque N2 est inanimé, et un accord au pluriel lorsque N2 est un animé humain. Si l'on en croit les nombreuses occurrences récoltées dans le British National Corpus, il semble d'ailleurs qu'il s'agisse là d'une tendance générale, bien que cette tendance ne puisse être érigée en règle unique et absolue. C'est ce que montrent les exemples de la catégorie suivante.

- le mouvement:

(36) A convoy of vintage Rolls-Royce cars has set out on a nostalgic journey. (BNC

K17 1769)

(37) Two Alton cousins, Christian Browning and Tom Marks are on their way to Romania with a convoy of aid workers, who are to help with orphanage in Gaesti.

(BNC B03 257)

Ce couple d'énoncés tend à première vue à confirmer le principe énoncé plus haut, selon lequel les N2 animés, contrairement aux inanimés, donneraient lieu à un accord au pluriel. On remarque toutefois, dans le second exemple, la présence d'un facteur supplémentaire permettant d'expliquer l'alternance singulier-pluriel: la seconde prédication (contenant l'accord au pluriel) correspond à une subordonnée relative en who , pronom qui est séparé de son antécédent par une virgule. Dans ce cas, il semble que l'antécédent de who ne soit plus constitué de l'ensemble N1 of N2, mais du seul N2. Autrement dit, il y aurait ici une sorte de dissociation du tout et de ses composants, ces derniers étant les seuls concernés par la deuxième prédication. Bien qu'il ne s'agisse pas du cas de figure le plus fréquent, une telle dissociation pourra se retrouver dans d'autres exemples, quelle que soit la catégorie à laquelle ils appartiennent.

- disposition verticale ou horizontale :

Cet ensemble de termes est, quant à lui, davantage compatible avec les inanimés qu'avec les animés humains.

On retrouve toutefois une alternance d'accords au singulier et au pluriel, alternance qui peut à première vue sembler contredire la tendance évoquée plus haut (réservant de préférence le pluriel aux animés).

Observons ces deux exemples, le premier suscitant un accord au singulier et le second un accord au pluriel :

(38) The track, after leaving the ridge, went steeply down for a few yards and then turned to the right and ran diagonally across the hill for a hundred yards; the pile of rocks was about midway on the right-hand side of this length of the track. (BNC BMY 1613)

(39) Sister Cooney picked up a pile of books and began to replace them on the shelves. (BNC A7J1906)

Dans le premier énoncé, l'accord au singulier est attendu. Dans le second, l'accord au pluriel ne l'est pas, mais n'invalide finalement pas le principe général évoqué plus haut, 
car le pronom them ne reprend pas ici l'ensemble $N 1$ of N2, mais le seul N2. Il s'avère que la totalité de ce GN est ici dissociée, et les parties (autrement dit les livres) sont en fait reprises pour être ré-agencées différemment.

- critères de sélection qualitatifs :

Avec ce type de termes, il faudra tout particulièrement examiner si l'accord est possible avec N1 ou avec le seul N2. En effet, la valeur qualitative du N1 peut éventuellement bloquer l'accord avec ce premier terme. On peut ainsi faire contraster:

(40) It may be that a variety of different approaches is considered appropriate. (BNC FTY 898)

(41) A variety of opinions were aired on the matter by the politicians present, and there seemed to be a definite acknowledgement that at least some of those present held shares in Anglo United, the mother company of IBM. (BNC CDD 291)

Dans le premier exemple, les aspects qualitatifs inhérents au terme variety ne font pas obstacle au fait de considérer ce terme comme sujet du prédicat be appropriate, dans la mesure où ce prédicat lui-même se caractérise par une dimension appréciative (et donc qualitative).

Dans le second exemple, en revanche, le prédicat n'est plus d'ordre appréciatif : ce n'est plus un verbe d'état mais un verbe d'action: il s'agit, plus précisément, d'un verbe de dire. De fait, l'accord avec le terme variety est rendu plus difficile car, notionnellement, ce n'est pas la variété qui est exprimée, mais ce sont les opinions. Ces dernières sont en outre variées: ainsi, sur le plan notionnel, il s'avère que le N1 fonctionne davantage comme un qualificatif que comme noyau du groupe nominal.

\section{Les noms de compagnies et les sigles}

En ce qui concerne les questions de concordance ou discordance entre morphologie et syntaxe, un autre ensemble de cas très particuliers est celui des noms assimilables à des noms propres et référant à des entreprises, compagnies, industries ou magasins. Nous verrons dans un second temps que les sigles présentent le même type de fonctionnement.

Parmi les noms de compagnies, on relève tout d'abord des cas d'accords faisant coïncider morphologie et syntaxe. Ainsi:

(42) Founded in 2001, Rotech already has a base in Houston and is also hoping to gain a presence in Australia, Dubai and Brazil. (The Scotsman)

Plus surprenants en première lecture, mais tout à fait explicables, nous trouvons des exemples tels que :

(43) Barclays has already put out its writedown for losses on securities linked to subprime in the States, and that, at $£ 1.3$ billion, was much better than feared. It remains to be seen whether RBS will have sidestepped those problems as well as its rival." (The Scotsman)

Il ne s'agit pas ici d'un vrai pluriel, mais d'un nom de compagnie portant une terminaison en -s. Il en existe de nombreux cas en langue anglaise (ex : Selfridges, Harrods) et l'on peut supposer que cette terminaison provient d'un génitif en -'s qui aurait été « raccordé » au nom, hypothèse qui permettrait donc de rapprocher de tels termes d'expressions comme at the butcher's. Quoi qu'il en soit, nous avons ici affaire à un terme au singulier, et il est donc logique que l'accord s'effectue au singulier. 


\section{coïncidences :}

- le premier cas de figure s'apparente au cas des noms collectifs, car on a affaire à un nom de compagnie au singulier, suivi d'un verbe au pluriel. C'est ce qu'illustrent les deux exemples ci-dessous:

(44) Olivant, the private equity group led by former Abbey chief Luqman Arnold, are not set to re-enter the race to take-over Northern Rock. (The Scotsman)

(45) Halifax Bank of Scotland have revealed plans to cut the interest rate on their current accounts despite promises to give customers ' 30 times the Royal Bank of Scotland' rate. (The Herald)

Les énoncés illustrant ce type de non-coïncidences étant très fréquents dans la presse, ceci montre bien qu'il ne s'agit pas d'erreurs de frappe. Dans de tels cas, la noncoïncidence entre morphologie et syntaxe s'explique en réalité de la même manière que pour les noms collectifs : il s'agit encore une fois d'accords sylleptiques, autrement dit, d'accords en fonction du sens et non de la morphologie. Ainsi, dans nos exemples, Olivant est un groupe et peut à ce titre être saisi sous l'angle de ces composants.

Dans le cas de Halifax Bank of Scotland, il s'agit d'une banque, et la décision annoncée émane de la direction de cette banque. Par le biais de l'accord au pluriel, l'énoncé souligne le fait que la direction est constituée d'un ensemble de personnes.

- le second type de non-coïncidences est à première vue très surprenant, car un nom au pluriel donne lieu à un accord au singulier. Ainsi:

(46) Forth Ports has slashed the value of its stake in Edinburgh Ocean Terminal but is to maintain its freeze on major land sales. (The Scotsman)

(47) For the third year running, Lloyds TSB Corporate Markets has won the Real FD/CBI FD's Corporate Bank of the Year award. It's given not only in recognition of our outstanding service, but also our ability to understand each customer's individual needs. Thank you to Britain's FD's who voted for us.(Lloyds TSB)

60 (48) Standard Life Investments is to stop investing in airlines through its Socially Responsible Investment (SRI) range of funds. (The Scotsman)

Une première explication peut être donnée par analogie. L'on sait en effet qu'en langue anglaise, il existe un certain nombre de termes dont la morphologie au pluriel donne toutefois lieu à un accord au singulier. Il s'agit de termes évoquant des noms de jeux, tels que draughts, darts, dominoes ou billiards. Il s'agit également de termes évoquant des noms de maladies: measles, mumps, rabies, ou encore les termes barracks, customs, gallows. Encore une fois, il est possible de faire appel au principe de l'accord sylleptique dans une version toutefois inverse de celle étudiée précédemment - afin d'expliquer de tels phénomènes. Ainsi, dans le cas de darts ou dominoes, c'est l'ensemble constituant le jeu qui prime lors de la saisie référentielle. Dans le cas de barracks, c'est le résultat du groupement de ces bâtiments, à savoir la caserne elle-même, qui est mis en évidence.

En ce qui concerne les deux exemples ci-dessus, faisant intervenir des noms de compagnies, on peut bien entendu faire appel à la même explication de saisie unitaire du référent, mais il semble en outre qu'une seconde explication puisse être énoncée, afin de compléter la première. Cette seconde explication fait intervenir une comparaison avec le fonctionnement des noms propres. Un nom propre se caractérise en effet par le fait qu'il est mono-référentiel ${ }^{9}$. De fait, et puisqu'il renvoie à un référent unique, il ne possède aucune signification, ni aucune définition. Autrement dit, le signe linguistique renvoie à son référent sans médiation, ce qui signifie que la division du signe linguistique en un signifiant et un signifié n'est plus pertinente. Pour cette raison, dans les cas où un nom

Corela, 6-2 | 2008 
propre ou nom assimilable est composé de plusieurs termes, il ne peut plus être considéré comme la somme des significations de ces termes, et ne suscite pas d'accord en genre et en nombre avec ses composants. C'est ainsi que l'on explique l'accord au singulier de Forth Ports, Lloyd TSB Corporate Markets ainsi que Standard Life Investments dans les exemples ci-dessus. En effet, il n'est plus ici question de ports, markets ou d'investments : ces termes ne sont pas à isoler, mais forment un tout indissociable avec les termes qui les précèdent. Chaque groupe réfère alors à la manière d'une étiquette, ou encore d'un nom propre, c'est à dire qu'il y a une référence directe au référent extralinguistique (en l'occurrence, les compagnies), référence qui n'est pas médiatisée par le biais de la signification. De fait, sur le plan référentiel, le tout n'équivaut donc pas à la somme de ses parties.

Examinons maintenant le cas des sigles. On observe, cette fois encore, une relative liberté d'accord, selon le point de vue adopté par l'énonciateur.

Dans un certain nombre de cas, il y accord en nombre avec le nom auquel renvoie le sigle. Ainsi:

(49) CBI calls for corporation tax reform. (The Scotsman- title)

Ici, CBI signifie Confederation for British Industry, et il y a donc coïncidence entre l'accord verbal et le singulier du noyau du groupe nominal, autrement dit confederation.

Très fréquemment, on observe toutefois un décalage entre l'accord verbal et le nom auquel le sigle fait référence. Deux facteurs expliquent de telles non-coïncidences :

- le fonctionnement de l'abréviation peut s'avérer similaire à celui d'un nom propre, lorsque l'accord se fait au singulier, tandis que le nom auquel il réfère est au pluriel.

(50) One city source appeared to confirm S\&N's suspicions over Carlsberg's motives, saying: "Many believe the major attraction of S\&N to Carlsberg is full control of BBH. Its weak spot, however, might be it wants to look at S\&N's books but is not willing to let shareholders see BBH's books." (The Scotsman)

Dans cet exemple, S\&N représente Scottish \& Newcastle et BBH tient pour Baltic Beverage Holdings. Nous voyons ici que l'accord du sigle se fait au singulier, relativement au signe lui même et non au sens.

(51) $\underline{H I E} \underline{\text { is }}$ to focus on local businesses with the highest ambitions and potential for growth. (The Scotsman)

L'accord s'explique ici de la même manière que précédemment. Tandis que HIE signifie Highlands and Islands Enterprises, groupe nominal comportant un noyau au pluriel, l'accord est au singulier. Il est relatif au sigle lui-même, considéré en tant que signe.

- l'accord peut être sylleptique, autrement dit tenir compte des composants existant dans l'extralinguistique. On observe alors des accords au pluriel, alors que le sigle renvoie à un nom au singulier :

(52) On justice, she claimed: "The SNP have achieved the incredible feat of even making Scottish Labour seem more in touch with public opinion." (The Scotsman)

Le sigle SNP signifie Scottish National Party, et l'accord au pluriel témoigne du fait que le parti est considéré sous l'angle de l'ensemble des personnes qui le composent.

Il en va de même dans l'exemple suivant :

(53) RSPB Scotland have pooled their resources as they prepare to give evidence against Donald Trump's $£ 1$ billion golf development plan in Aberdeenshire. (The Scotsman) 
71 Sachant que RSPB signifie Royal Society for the Protection of Birds, on retrouve ici le même principe d'accord au pluriel d'une entité collective.

\section{Les épicènes}

Un épicène se définit comme un terme qui ne varie pas morphologiquement, qu'il soit au masculin ou au féminin. Des exemples classiques d'épicènes en langue anglaise sont child ou encore teacher. Corrélativement - et cette tendance est de plus en plus marquée dans les pays anglo-saxons - l'accord pronominal (ou celui de l'adjectif possessif) d'un épicène peut s'effectuer par le biais du pluriel, qui est non marqué en genre, et ce afin d'éviter l'emploi d'un pronom singulier qui, lui, est nécessairement marqué en genre. Ce fonctionnement permet d'éviter les reprises par he or she, qui sont peu économiques et tendent à alourdir le style de l'énoncé, comme on le voit dans l'exemple suivant :

(54) If the black child is placed in an environment which is hostile, the inevitable consequence of this will be repeated attacks on the child's personality which will cause severe damage to his or her self-esteem. (BNC CRW675)

Les reprises des épicènes par un pronom ou adjectif possessif au pluriel permettent donc d'expliquer certains exemples qui peuvent, à première vue, sembler incongrus, tels que :

(55) Lock a Brit in an over-crowded Third World prison and there are certain things they will miss even more desperately than a chastity belt. (The mirror)

Dans cet exemple, la reprise de a Brit par they est bien entendu assez surprenante pour un francophone, mais cette convention permet cette fois encore d'éviter la reprise de a Brit par he or she.

Le même phénomène est à l'œuvre avec un certain nombre d'épicènes, dont nous proposons ici une liste non exhaustive :

- child:

(56) What age is a child when they lose their first tooth? (BNC)

\section{- friend:}

(57) What to do when your friend loses their job. (BNC)

- person:

(58) "My job is to make sure the person who loses feels like they have been treated fairly so that their supporters will support the winner," Dean told The Associated Press. (USA Today)

- teacher:

(59) A teacher who suspects that a colleague has acted improperly towards a pupil must not be deterred from disclosing their concern because of fears about the effect on employment prospects. (BNC HX 14468)

- leader:

(60) The third front-runner, Iain Gray, who is believed to be favoured by Gordon Brown, has added that the new leader will have "a powerful mandate" of their own. (The Scotsman)

\section{- donor:}

(61) Currently patients receiving blood transfusions are at risk of contracting the disease from a donor who does not even know they are infected. (USA Today)

- member:

(62) Every member of the Labour party carries with them a simple guiding mission on the membership card. (The Guardian) 
Cet exemple s'avère particulièrement intéressant, car il montre que la reprise au pluriel ne concerne pas le prédicat: elle s'applique uniquement aux adjectifs possessifs ou, comme ici, aux pronoms personnels. Ceci s'explique aisément : le prédicat, contrairement aux adjectifs possessifs ou pronoms, n'est en aucune façon porteur d'indications de genre. Il n'est donc pas nécessaire d'accorder le prédicat au pluriel.

Ajoutons un autre exemple, qui peut à première vue sembler tout à fait incongru :

- soldier:

(63) "If a soldier loses their helmet, they pay for it. If they get blown up and lose their helmet, they get billed for it." (The Guardian)

Dans cet exemple, le calcul référentiel est peut-être moins aisé que dans les cas précédents, car il faut ici éviter le piège des idées reçues : en effet, un soldat n'est pas nécessairement du sexe masculin, et le terme soldier fonctionne bel et bien en tant qu'épicène.

Cette fois encore, l'emploi du pluriel permet donc d'éviter l'emploi d'une formule telle que his or her.

Plus difficilement explicable, cependant :

- partner :

(64) Can you sue your partner if they get pregnant without your permission? (The mirror)

79 Il est vrai que le terme partner est théoriquement un épicène (il n'existe pas un terme morphologiquement différent au féminin). Toutefois, le champ des possibles semble ici restreint co-textuellement par le prédicat get pregnant qui est jusqu'à présent réservé aux femmes. Mais peut-être l'exemple devance-t-il les avancées de la génétique?

Remarquons par ailleurs que les indéfinis tels que someone, anyone, ou everyone sont eux aussi généralement repris par des pronoms personnels ou adjectifs possessifs au pluriel, bien qu'ils déclenchent des accords verbaux au singulier. Ainsi:

(65) I believe in a Scotland where everyone should have the chance to rise as far as their talents can take them, where the talents of each of us should contribute to the wellbeing of all and I believe that's what most people in Scotland also believe. (discours de G. Brown-28-03-08)

81 Notons enfin qu'une alternative existe, dans le domaine de la reprise par des épicènes. Ainsi, si la majorité des reprises s'effectue par le pronom they, il n'est pas non plus impossible de trouver le pronom she valant pour he or she. Ainsi:

(66) We need a Scotland that cares for the weak and powerless, especially the unborn child who is ripped from the womb and slaughtered before she even has a chance to speak. (The Scotsman)

Nous voyons ici que la référence est générique, si bien qu'une reprise par le pronom they aurait pu éviter le choix du féminin.

Ce type de reprise par she semble d'autant plus aisé que le terme employé est child, ainsi qu'en témoigne ce second exemple :

(67) On the basis of this analysis, a child with severe learning difficulties might have both delayed language, in that she would be functioning at a level below that normally expected for children of her age, and a deficit if her language skills were more severely affected than other areas. (BNCCG6 479)

84 Ce phénomène, étant récurrent, concurrence ainsi la reprise par un pluriel. Cette dernière reste toutefois la plus fréquente. 


\section{Les cas d'ellipses} crews est possible? Il s'avère que le pluriel crews réfère à des équipages, tandis que dans nos exemples, il est question de membres d'équipage. Nous émettons donc l'hypothèse que ces exemples résultent de l'ellipse du terme member qui, lui, aurait pu porter la marque du pluriel. Cette ellipse étant effectuée, il est alors préférable de ne pas accorder crew dans le cas où l'on réfère à des membres de l'équipage: l'accord pourrait, en première lecture, susciter des confusions (membres ou équipages?) Par le biais de l'absence d'accord, c'est donc un principe de différenciation qui est mis à l'œuvre.

91 On remarque que ce type de fonctionnement se retrouve à l'identique dans les cas de staff :

(73) Two staff - Colin McKenzie, also of Stakis Highland Lodges, and Vincent di Nozzi of the Dunblane Hydro -received 20-year service awards of a gold watch plus a gold, emerald and diamond pin. (BNC A7F 850)

(74) Mr Justice Brooke ruled that Northumberland health authority should have specified that three staff were necessary. (BNC A50 284)

Le cas du terme personnel est également similaire :

(75) The C\&P site at Baleycourt, for instance, put in 24 entries from just $\underline{100}$ personnel. (BNC K9K 7)

(76) The number of US personnel in the USAID office in Yemen was reduced from 19 to one before the outbreak of the war on Jan. 15, with the remaining official scheduled to leave by Jan. 30, and local staff were given" administrative leave" as of Feb. 2 . (BNC HL4 2390)

Notons toutefois le phénomène suivant : dans le cas où l'un ou l'autre de ces termes est précédé d'un adjectif numéral, non plus cardinal mais ordinal, il semble que le 
rétablissement du terme member soit une constante (aucun contre-exemple n'ayant été trouvé dans le British National Corpus), à l'image de l'exemple suivant :

(77) The navigator had survived with head injuries but the third crew member was

O.K. I signalled to Athens asking them to designate the nearest airfield where I could pick up Brown and White in a Maryland. (BNC CA8 748)

Ceci s'explique d'ailleurs fort bien car, en l'absence d'accord requis par l'adjectif ordinal, il pourrait alors y avoir ambiguité (membre ou équipage entier ?). Le rétablissement du terme member semble donc répondre à un principe de désambiguïsation.

\section{Les cas de métonymie}

Dans certains énoncés, les phénomènes de discordance entre morphologie et syntaxe semblent pouvoir être expliqués par des cas de métonymie ${ }^{10}$. Cette substitution a lieu entre termes contigus à l'intérieur d'un même ensemble logique structuré en parties. Ainsi :

(78) Michael Vaughan admitted England's 'confused' selection policy led to their Headingley hammering. (The Sun)

Dans cet exemple, England désigne en réalité l'équipe de cricket d'Angleterre : il s'agit donc d'un cas de métonymie, par substitution du terme désignant la nation au terme référant à l'équipe elle-même. L'accord s'effectue en fonction du sens, autrement dit en tenant compte des différents membres constituant cette équipe.

Prenons cet autre exemple :

(79) France took a big decision some years ago to tackle their own rising rates of liver disease. They completely banned advertising of alcohol and put controls on when and where it was available. (The Scotsman)

Ici, France réfère en réalité au gouvernement français, ce qui justifie alors la reprise par un pronom personnel au pluriel. Ces exemples montrent bien que les raisons donnant lieu à une discordance entre morphologie et syntaxe sont multiples. Parler d'accord sylleptique n'est pas nécessairement une justification suffisante, et bon nombre de cas requièrent l'identification de la cause de cet accord sylleptique.

\section{L'emploi de marqueurs et expressions partitives ou totalisantes}

Le cas de certains marqueurs ou expressions permettant soit de prélever, soit de regrouper des éléments est également à examiner.

Envisageons tout d'abord le cas du pronom which. Il est employé lorsque l'énonciateur demande au co-énonciateur de prélever et désigner un ou plusieurs éléments. Puisque la quantité à prélever peut être plurielle mais indéfinie, l'emploi de which peut donc susciter un accord au pluriel, comme dans l'exemple suivant :

- which:

(80) Which of the family are wearing something green? (BNC BNG 563)

Un phénomène similaire s'observe dans le cas d'expression quantificatrices :

- a large number: 
(81) The computer did not plan an Agnes or a Paul, but only a prototype known as a human being, giving rise to a large number of specimens which are based on the original model and haven't any individual essence. (BNC ABS 1735) constituant la tête du groupe nominal a large number of specimens, n'entraîne toutefois pas un accord au singulier, comme le voudrait théoriquement la règle. Deux explications semblent possibles :

- soit l'accord se fait avec le nom le plus proche du prédicat, à savoir le N2 de cette structure N1 of N2,

- soit l'accord s'effectue selon un fonctionnement de type "accord sylleptique», dans la mesure où l'expression a large number of - en dépit de sa forme au singulier - évoque en réalité une pluralité d'éléments.

Prenons un second exemple, faisant cette fois intervenir un N2 présentant un fonctionnement de type " nom collectif » :

(82) I think that does give such programmes genuine mass appeal when you have ordinary people talking about their gardens - not huge grand gardens, quite often the kind of suburban gardens that a large number of the audience are familiar with. (BNC ATA 98) catégorie de fonctionnement du nom qui suit.

Prenons un exemple ordinaire, dans lequel the whole est suivi d'un nom discret au singulier :

(83) When too many snap, the whole web collapses into a sticky mess. (BNC CM4 3184)

L'accord s'effectue ici au singulier, conformément aux attentes suscitées par les règles syntaxiques. Tel n'est toutefois pas le cas de l'exemple suivant:

(84) The whole bridal party are expected to arrive about ten minutes before the ceremony. (BNC BPF341) Ici, l'accord s'effectue au pluriel, autrement dit il s'effectue avec le terme party qui présente - nous l'avons montré plus haut - un fonctionnement de type " nom collectif ».

107 Si cet accord s'explique par le fait que le nom-tête du groupe nominal est constitué par party, l'accord devient en revanche plus surprenant dans l'exemple suivant :

(85) The whole of the party were in excellent health and had well tried each others stamina in walking up mountains etc. etc. but having so many interesting objects in view they had allowed no difficulty or danger to impede their progress. (BNC HRB 932)

Cette fois, le nom-tête du groupe nominal est whole: contrairement à l'exemple précédent, dans lequel whole jouait un rôle adjectival, il est ici nominalisé, et il semblerait donc naturel que l'accord s'effectue au singulier. Il n'en est rien, ce qui montre que les règles syntaxiques ne sont pas nécessairement mises en application. 


\section{- the majority of:}

(86) The majority of the fry are eaten but I think that this is a case of survival of the fittest in the confines of a community tank. (BNC C97 2630)

Une fois encore, on aurait pu s'attendre à un accord au singulier. Ici, contrairement à ce qui se produit dans le cas de l'expression a large number citée plus haut, il s'avère que l'expression a/ the majority, lorsqu'elle constitue le noyau du groupe nominal, est d'ordinaire suivie d'un singulier. Il n'est est rien dans cet exemple, et la raison semble en être la nature de la complémentation (fry), dont nous avons montré qu'elle appartient à la catégorie des noms collectifs. Il semble donc que l'accord se fasse sous l'influence de ce N2.

111 Cet exemple témoigne donc, encore une fois, de l'importance de la signification dans les phénomènes d'accord en langue anglaise, la morphologie étant alors reléguée au second plan.

\section{Conclusion}

Cette étude a permis de recenser un certains nombre de phénomènes relevant tous de ce que l'on peut qualifier d'«accord sylleptique». Nous avons toutefois montré que les raisons de cet accord sylleptique peuvent être multiples, et il importe donc de les identifier, afin d'apprécier les phénomènes dans leur singularité. Ce qui ressort de cette étude est également une certaine flexibilité de la langue anglaise qui, davantage que le français, permet de se libérer des contraintes syntaxiques pures afin de privilégier le sens. En outre, les accords extrêmement fluctuants caractérisant l'emploi de certains termes doivent nous rappeler que la langue n'est pas un code, que la subjectivité de l'énonciateur est toujours (de façon plus ou moins discrète) à l'œuvre, et qu'un grand nombre de phénomènes linguistiques sont dépendants du cotexte, ce qui signifie également qu'ils sont explicables par celui-ci.

\section{BIBLIOGRAPHIE}

Arigne V. (2006), « Les discrets collectifs face aux massifs : des modes de discrétisation des noms massifs », Cycnos (en ligne), vol. 23, nº 1, Le Qualitatif.

Arigne V. (1998), « Le nom : problèmes de nombre et catégorisation nominale en anglais », in Mélanges Jean Tournier, Recherches en linguistique étrangère $n^{\circ} \mathrm{XIX}$, Annales Littéraires de l'Université de Franche-Comté.

Borillo, A. (1996), « Statut et mode d'interprétation des noms collectifs », in Cl. Guimier (éd.) 1996, Cotexte et calcul du sens, Presses universitaires de Caen, p. 105-121.

Bouchard Denis (1995), The Semantics of Syntax. A Minimalist Approach to Grammar, University of Chicago Press.

Corela, 6-2 | 2008 
Cruse, D.A. (1986), Lexical Semantics, Cambridge, Cambridge University Press.

Culioli A (1991), Pour une linguistique de l'énonciation. Opérations et représentations, Tome 1, Coll. L'Homme dans la langue, Paris, Ophrys.

Depraetere, I. (2003), “On verbal concord with collective nouns in British English”, in English Language Linguistics, vol. 7, part 1, Cambridge, Cambridge University Press, p. 85-127.

Gillon, B.S. (1992), “Towards a common semantics for English count and mass nouns”, Linguistics and Philosophy, 15, p. 597-639.

Jespersen, O. (1924) The Philosophy of Grammar, London, George Allen \& Unwin.

Kleiber, G. (1997) « Massif/ comptable et partie/ tout », in Verbum, XIX, 3.

Lyons, J. (1977), Semantics, Cambridge, Cambridge University Press.

Simons, P. (1987), Parts, a Study in Ontology, Oxford, Clarendon Press.

Vaxelaire J-L. (2005), Les Noms Propres. Une analyse lexicologique et historique, Paris, Champion.

Wierzbicka, A. (1988) The Semantics of Grammar, Amsterdam/Philadelphia, John Benjamins Publishing Company.

\section{Grammaires citées :}

Galiban J.P. (2006), Grammaire expliquée de l'anglais, coll. Optimum, Paris, Ellipses.

Berland-Delépine S. (1990), Grammaire pratique de l'anglais, coll. Enjoy your Grammar, Paris, Ophrys.

Adamczewski H. (1993), Les clés de la grammaire anglaise, Paris, Armand Colin.

Berland-Delépine S. (2006), Butler R. Grammaire anglaise de l'étudiant, coll. Enjoy your Grammar, Paris, Ophrys.

Hamilton S. (1999), Grammar in action, Yvelines, Spratbrow.

Faudon B. (2008), La grammaire et l'usage de l'anglais, coll. Optimum, Paris, Ellipses.

\section{NOTES}

1. Citons notamment Grammaire expliquée de l'anglais, éds. Ellipses ; Grammaire pratique de l'anglais, éds. Ophrys; Les clés de la grammaire anglaise, éds. Armand Colin ; Grammaire anglaise de l'étudiant, éds. Ophrys ; Grammar in action, eds. Spratbrow ; La grammaire et l'usage de l'anglais, éds. Ellipses.

2. Les grammaires se contentent généralement de mentionner les phénomènes en question, sans davantage d'explications. Prenons l'exemple de Grammaire expliquée de l'anglais, qui annonce « Noms singuliers désignant un ensemble de gens avec accord pluriel » et qui en dresse une liste suivie de " etc....»

3. A. Culioli, Pour une linguistique de l'énonciation. Opérations et représentations, Tome 1, Coll. L'Homme dans la langue, Paris, Ophrys, 1991.

4. Les parenthèses encadrant le symbole Qnt signifient qu'il y a une prépondérance du qualitatif.

5. On notera que ce terme est parfois orthographié avec une majuscule.

6. Rappelons que l'hyponymie est la relation sémantique d'un lexème à l'autre, selon laquelle l'extension de l'un est incluse dans l'extension de l'autre. 
7. Rappelons qu'une relation de méronymie est une relation de partie à tout. Un méronyme $A$ d'un terme B est un terme dont le signifié désigne une sous-partie du signifié de B (inversement, il serait possible de qualifier B d'hyperonyme de A).

8. V. Arigne, «Les discrets collectifs face aux massifs : des modes de discrétisation des noms massifs ", Cycnos (en ligne), vol. 23, n 1, Le Qualitatif , Février 2006.

9. Pour davantage de précisions, voir J-L. Vaxelaire, 2005, Les Noms Propres. Une analyse lexicologique et historique, Paris, Champion.

10. Rappelons que l'on parle de métonymie lorsqu'un concept est dénommé à partir d'un mot désignant un autre concept.

\section{RÉSUMÉS}

Cette étude s'intéresse aux phénomènes de discordance entre morphologie et syntaxe, tel l'accord verbal au pluriel de noms morphologiquement au singulier, de même que le cas inverse. Une explication en termes d'accord sylleptique, bien que juste, s'avère toutefois insuffisante face à la variété des phénomènes concernés, et il faut systématiquement s'intéresser à la cause de cet accord sylleptique. Outre le cas des noms collectifs (dont nous proposons une typologie), nous nous intéressons à l'accord suscité par les expressions de type N1 of N2, dans lesquelles N1 évoque une multiplicité d'éléments, ainsi qu'au cas des noms d'entreprises ou de compagnies. L'étude montre également qu'un certain nombre de discordances morpho-syntaxiques s'explique par des phénomènes de métonymie, d'ellipse ou encore par l'emploi de noms épicènes. Certaines expressions quantificatrices s'avèrent également surprenantes par les accords fluctuants qu'elles sont à même de susciter, et nous cherchons donc à expliquer ces alternances d'accords au singulier et au pluriel.

This paper examines phenomena of discordance between morphology and syntax, such as the plural concord of verbs with nouns which are morphologically in the singular, or vice versa. The principle of sylleptic agreement, which is used to account for such phenomena, is not sufficient to describe the great variety of cases, and it is thus necessary to identify the reasons for those sylleptic agreements. Besides the case of collective nouns (for which we propose a typology), this study deals with the concords that take place with N1 of N2 expressions, in which N1 evokes a multiplicity of elements, and it also examines the concords characterizing nouns of firms or companies. This paper also shows that a certain number of mismatches between morphology and syntax can be explained thanks to phenomena of metonymy, ellipsis or thanks to the use of epicene nouns. Some expressions used to express quantification also lead to surprising concords, which can either be in the singular or in the plural according to the context, and this study tries to account for the possibility of such alternations.

\section{INDEX}

Mots-clés : accord sylleptique, nom collectif, métonymie, ellipse, épicène

Keywords : sylleptic agreement, collective noun, metonymy, ellipsis, epicene Noun 
AUTEUR

BLANDINE PENNEC

Université de Perpignan, Laboratoire LIDILE, EA3874, Université Rennes 2 